\title{
Necrotizing fasciitis: A review of three clinical encounters
}

\author{
Jaron L. Pettis, Neelam Mulji, Fernando A. Navarro* \\ University of South Carolina School of Medicine, Columbia, SC, United States
}

Received: September 26, 2018

Accepted: November 23, $2018 \quad$ Online Published: November 29, 2018

DOI: $10.5430 /$ css.v5n1p5

URL: https://doi.org/10.5430/css.v5n1p5

\begin{abstract}
Background: Necrotizing fasciitis is a potentially lethal soft tissue infection characterized by rampant necrosis and destruction of subcutaneous tissues. Current estimates of necrotizing soft tissue infections in the United States are 4.3 infections per 100,000 of the population. Although the incidence of necrotizing soft tissue infections has decreased in the last decade, the toxic and lethal nature of the disease process lends utmost importance to accurate diagnosis and immediate management. The purpose of this review article is to report three cases of necrotizing fasciitis and provide literature review in regards to hallmark characteristics, predisposing risk factors and treatment optimization.

Case: The first case depicts a newly diagnosed 43-year-old male HIV patient with necrotizing fasciitis infection characterized by Klebsiella, Serratia and anaerobic bacteria cultures. The second case describes the course of a 71-year-old male diagnosed with necrotizing fasciitis in the setting of a complicated anal fistula characterized by B. fragilis, S. anginosus and Prevotella species. The third and final case describes the course of a 44-year-old female diagnosed with necrotizing fasciitis in the setting of Ludwig's Angina characterized by Klebsiella and Dubliensis species. Treatment was initiated with extensive wound debridement, multiple washouts and broad antibiotic regimens in all three cases. Additional hyperbaric oxygen therapy was administered in the third case.

Conclusions: These case reports illustrate the range of severity and settings in which necrotizing fasciitis can occur. Significant morbidity and mortality rates are associated with a delay in treatment initiation. Given this, it is of utmost importance to develop and maintain a high clinical acumen for necrotizing soft tissue infections.
\end{abstract}

Key Words: Necrotizing fasciitis, Hyperbaric oxygen, Ludwig angina, Surgical debridement, Review, Soft tissue infection

\section{BACKGROUND}

Necrotizing fasciitis is a potentially lethal soft tissue infection characterized by rampant necrosis and destruction of subcutaneous tissues. Infections are classified according to the layers of tissue that are affected, including skin, fascia and muscle. Current estimates of necrotizing soft tissue infections in the United States are 4.3 infections per 100,000 of the population. ${ }^{[1]}$ Although the incidence of necrotizing soft tissue infections has decreased in the last decade, the toxic and lethal nature of the disease process lends utmost importance to accurate diagnosis and immediate management. The purpose of this review article is to report three cases of necrotizing fasciitis and provide literature review to this disorder regarding hallmark characteristics, predisposing risk factors and treatment optimization.

Necrotizing fasciitis is a subtype of necrotizing soft tissue infections confined to the superficial fascia. Pathogenesis is marked by the inoculation of bacteria into subcutaneous tis-

\footnotetext{
*Correspondence: Fernando A. Navarro; Email: fernando.navarro@uscmed.sc.edu; Address: University of South Carolina School of Medicine, Columbia, SC, United States.
} 
sues via any break in the epithelial or mucosal surfaces. Methods of inoculation include catheter insertion sites, trauma, injections, ulcers, abscesses, fistulas and even hematogenous spread from a distant site. The most commonly affected anatomic locations are extremities, perineum and genitalia. ${ }^{[2]}$

\section{Classification}

Infections can be classified according to their anatomic location, depth of infection and most importantly their microbial cause. Many academic sources utilize the microbial classification system organizing infections into Type I, II, III, and IV categories. Type I infections are usually polymicrobial in nature and comprise the majority etiology of necrotizing fasciitis infections. Often, immunocompromised individuals including the elderly, diabetics and chronic kidney failure patients are affected. Infections occur following trauma or surgical operations due to the patient population's altered healing potential. ${ }^{[3]}$ Sensationalized in the media as the "flesh-eating disease", Type II infections primarily comprise Group A streptococcus or Staphylococcus aureus microbes. While less common than Type I infections, evidence of community acquired methicillin-resistant Staphylococcus aureus (MRSA) suggest increasing incidence of Type II infections. These infections can occur in healthy populations across a range of ages, however most commonly follows a recent history of trauma or surgical operation. ${ }^{[3]}$ Type III infections involve the Aeromonas hydrophila and Vibrio vulnificus microbes in the setting of freshwater and seawater lesions, respectively. Clostridium perfringens is also placed in this category, identified by its destructive gas gangrene capacity. Physical exam findings of crepitus confirm involvement of this specific microbe. ${ }^{[3]}$ Finally, Type IV infections are dedicated to fungal organisms, in particular Candida. Even more rare than Type III infections, these occur in primarily immunocompromised individuals with traumatic wounds as well as burns. ${ }^{[3]}$

\section{Pathophysiology}

Necrotizing soft tissue infections arise in the subcutaneous tissue facilitated by open wounds, trauma or via spread from perforated viscera. Microbes infiltrate and traverse the superficial and deep fascial planes, accessing the deeper layers and structures (show diagram). Horizontal spread can also occur to a degree. Common sites of infection include the lower leg, upper limbs, perineum, trunk and head and neck. ${ }^{[4]}$

While causative bacteria utilize a variety of mechanisms - aerobic, anaerobic or facultative aerobic - all manifest their lethality through virulent toxins. Examples of such toxins include extracellular pyrogenic exotoxins A, B and C, mitogenic exotoxin $\mathrm{F}$ and streptococcal-specific exotoxins.
These toxins function as superantigens, interacting with and disrupting major histocompatibility complex (MHC) Class II molecules and T-lymphocyte receptors. This interaction leads to activation of large numbers of $\mathrm{T}$ cells and subsequent release of interleukins and inflammatory cytokines (i.e. tumor necrosis factor, gamma interferon). In this regard, microbes destroy human tissue via direct insult and blood supply compromise. ${ }^{[4]}$

The release of toxins and subsequent inflammatory cytokines results in destruction of the venous circulation. This fundamental feature of necrotizing soft tissue infections initiates the process of ischemia and ultimately necrosis and cell death. Toxins promote a hypoxic environment for microbes to survive. Rapid reproduction and proliferation of the bacteria and subsequent toxins enhances their spread into surrounding healthy tissue. ${ }^{[4]}$

\section{Clinical manifestations}

Early stages of pathology demonstrate normal-appearing skin. It is not until the infections accesses the deeper structures that clinical manifestations present. Symptoms begin within the first 24 hours of incitement and are categorized according to their time of onset. Wong and Wang propose three different stages of symptoms. Stage I features pain out of proportion, fever, extensive erythema, edema and calor. At this point in the disease process, there is difficulty in establishing a diagnosis of cellulitis versus a necrotizing soft tissue infection. ${ }^{[5]}$ Due to the similar initial presentations, it is challenging to discern the two until deeper involvement presents. The appearance of extreme local tenderness in addition to blistering, bullae formation and ulceration occurs in Stage II of the disease process. ${ }^{[5]}$ Microbes begin to affect the superficial nerves and deeper structures, manifesting as crepitus, skin anesthesia and generalized dusky discoloration secondary to necrosis in Stage III. ${ }^{[5]}$ The transition from Stage II to Stage III distinguishes the spreading nature of necrotizing soft tissue infections from the relatively confined cellulitis infections. Beyond the stages involve infiltration into other organ systems, most importantly the bloodstream. Patients develop toxic shock syndrome and septicemia. The body responds to systemic infection with hypotension, tachycardia and fever. Severe toxicity and death occur if not recognized and treated immediately. ${ }^{[5]}$

\section{Diagnosis}

The diagnosis of necrotizing fasciitis begins with a good clinical exam. Clinical findings of a patient with necrotizing fasciitis will show signs of local soft tissue infection such as erythema, edema, calor, and tenderness. Due the spreading nature of necrotizing fasciitis patients can also have systemic 
signs such as fever or hemodynamic instability. The site of infection may also present with a hard knob feeling that extends beyond the site of apparent skin involvement. Other classic signs for necrotizing fasciitis involve tissue crepitus (due to the tendency of the causative microbes to produce gas), along with the worrisome signs of, pain out of proportion to physical findings, and rapid deterioration of the patient. ${ }^{[6,7]}$ A scoring system has also been proposed by Wang et al. known as the Laboratory Risk Indicator for Necrotizing Fasciitis (LRINEC). This system uses the lab values of CRP, WBC, Hemoglobin, Sodium, Creatinine, and Glucose (see Table 1). A LRINEC score $>5$ puts a patient at moderate suspicion for necrotizing fasciitis and further testing modalities should be pursued to rule in or out necrotizing fasciitis, such as imagine modalities. ${ }^{[7]}$ The original article created by Wong et al. notes that values $\geq 6$ on the LRINEC score had a positive predictive value of $92 \%$ and a negative predictive value of $96 \% .^{[8]}$ This suggests that the LRINEC system can be a valuable tool for assessing for necrotizing fasciitis. Of note, a study conducted on 948 emergency room patients showed that patients with confirmed necrotizing fasciitis, 63.8\% were categorized as having a low LRINEC score $(<5){ }^{[9]}$ Therefore, while the LRINEC score system can be used to assist in guiding suspicion of necrotizing fasciitis, it is not a perfect system.

Imaging can be a useful tool for identifying necrotizing fasciitis in patients with a low clinical suspicion. However, due to the considerable consequence of delaying treatment, imaging should only be used in cases where the patient is not deteriorating rapidly or have the presence of crepitus. As these two symptoms would call for urgent surgical operation for treatment. Computed tomography is a common radiographic method used for evaluation of suspected necrotizing fasciitis. Signs to look for would be evidence of gas in soft tissue, fluid collections, and inflammatory changes beneath the fascia. ${ }^{[6,10]}$ Ultrasound is another study modality that can be performed quickly to detect abscess formation or the presence of gas formation in the fascial layer. ${ }^{[11]}$ However due to a lack of research with ultrasound CT still remains the primary imagine modality. Another potential imaging modality that can be used is MRI. MRI is known for its better resolution of soft tissue in comparison to CT. This enhanced image quality allows for visualization of fascial thickening from the infection confirming a diagnosis of necrotizing fasciitis. ${ }^{[12]}$ However, due to MRI having a higher sensitivity compared to specificity for identifying deep fascial involvement, it can lead to over estimation of the deep tissue involvement in necrotizing fasciitis. ${ }^{[13]}$ Along with overestimation of tissue involvement, imaging through MRI also takes more time to obtain than a CT scan. This results in a longer delay to the operating room for patients who have necrotizing fasciitis. Due to the nature of how necrotizing fasciitis functions any delays in time can result in significant adverse outcomes.

Table 1. Laboratory Risk Indicator for Necrotizing Fasciitis (LRINEC)

\begin{tabular}{|c|c|}
\hline Test & Score \\
\hline \multicolumn{2}{|l|}{ CRP (mg/dl) } \\
\hline - $<15$ & 0 \\
\hline$\bullet \geq 15$ & 4 \\
\hline \multicolumn{2}{|c|}{$\mathrm{WBC}(\times 100,000 / \mu \mathrm{l})$} \\
\hline$\bullet<15$ & 0 \\
\hline - $15-25$ & 1 \\
\hline - > 25 & 2 \\
\hline \multicolumn{2}{|c|}{ Hemoglobin (g/dl) } \\
\hline • > 13.5 & 0 \\
\hline - $11-13.5$ & 1 \\
\hline$\bullet<11$ & 2 \\
\hline \multicolumn{2}{|c|}{ Sodium (mmol/L) } \\
\hline - $\geq 135$ & 0 \\
\hline$\bullet<135$ & 2 \\
\hline \multicolumn{2}{|c|}{ Creatinine (mg/dl) } \\
\hline$\bullet \leq 1.6$ & 0 \\
\hline • > 1.6 & 2 \\
\hline \multicolumn{2}{|c|}{ Glucose (mg/dl) } \\
\hline - $\leq 180$ & 0 \\
\hline • > 180 & 1 \\
\hline
\end{tabular}

Even with evidence from both the clinical exam and imaging modalities, the only way to definitively arrive at a diagnosis of necrotizing fasciitis is through surgical exploration. In the operating room the patient will have the wound incised and on inspection the operating surgeon will find swollen and grey fascia with areas of necrosis, exudates that are often brown in color, and tissue planes that can be dissected easily through blunt dissection, due to undermining of the surrounding tissue by the infection. Furthermore, samples should be collected and sent for culturing and sensitivity testing of the causative organism. ${ }^{[6]}$

\section{Treatment}

If there is strong clinical suspicion of necrotizing fasciitis then urgent surgical exploration is indicated. Signs that help raise the suspicion of necrotizing fasciitis are hypotension, crepitance, skin necrosis, bullae, gas on imaging, WBC > $15.4 \mathrm{~K} / \mu \mathrm{l}$, and $\mathrm{Na}<135 \mathrm{mmol} / \mathrm{L}^{\left[{ }^{[14]}\right.}$ With the whole clinical picture suspicious of necrotizing fasciitis, surgical exploration is performed to both confirm the diagnosis and debride the involved tissue to the extent that is necessary. ${ }^{[15]}$ This 
debridement should take place as quickly as possible. Delays in debridement past twenty four hours after admission are associated with higher rates of mortality among patients with necrotizing fasciitis. ${ }^{[15]}$ Once the patient has been admitted to the hospital it is important to optimize the patients nutrition and fluid. With a patient who has necrotizing fasciitis it is best to admit them into an ICU setting where close monitoring of the patients vitals can be performed, and if needed aggressive fluid resuscitation and inotropic treatments can be initiated in the complication of septic shock. Oftentimes, these patients become critically ill and will require a central line placement, a foley catheter, and occasionally endotracheal intubation may become necessary. All of which increase the need for ICU care.

Since necrotizing fasciitis is from an infectious cause antibiotics are a necessary addition to treatment. Initially, antibiotics should cover a broad spectrum of organisms and be administered as early as possible. Anti ribosomal antibiotics, such as clindamycin, are also key in the treatment process as they negate gram positive organism that are amplifying the severity of the necrotizing fasciitis with exotoxin production. ${ }^{[16]}$ Once cultures begin to indicate which organisms are responsible, pharmaceutical treatment can be narrowed to cover the susceptibilities of the organisms present. Antibiotics should be continued as long as necessary pending on the area involved. Some areas will require longer treatments than others due to difficult penetration and treatment, such as osteomyelitis. Ultimately, antibiotics should be continued until the infection has been controlled, and administration should continue until local signs and symptoms of the infection have resolved for 5 days. To reach this point in treatment can take weeks with necrotizing fasciitis with an average hospital stay of 46 days in one study. ${ }^{[7,17]}$

Necrotizing fasciitis will require surgical debridement and as such the area of debridement should be aggressive. No consideration for reconstruction should take place initially as doing so could potentially compromise proper debridement margins. ${ }^{[2]}$ During the initial debridement, tissue samples should be taken and sent to pathology to be analyzed. This analysis will confirm the diagnosis of necrotizing fasciitis and help optimize pharmaceutical treatment for this patient. ${ }^{[18]}$ Once the debridement is completed the wound should be repeatedly and profusely irrigated with a broad spectrum antiseptic solution. ${ }^{[3]}$ On finishing up the initial debridement the wound should be covered with an antiseptic dressing. The patient at this time is returned to the ICU where they will undergo management of fluids and nutrition. Additionally, they will be continued on antibiotics and have their antimicrobial dressings changed as needed. Throughout the rest of the patients stay, they will return to the operating room for a second look operation and additional debridement surgeries as it often takes multiple debridements to gains source control. The second look surgery should take place within twenty-four hours of the initial debridement. It is not uncommon for patients to require multiple subsequent debridements throughout their hospital stay. The patient should return to the operating room at scheduled intervals to evaluate for continued spread of the infection until disease progression has stopped. In fact the average necrotizing fasciitis patient will undergo three to four subsequent debridement surgeries. ${ }^{[2,19,20]}$ Other common surgeries that take place in the setting of necrotizing fasciitis due to wound location are fecal diversion surgeries and amputations. Fecal diversion surgeries are helpful in the setting of a perianal or perineal region necrotizing fasciitis. This helps to divert the fecal contents and reduce secondary wound contamination risks. ${ }^{[21]}$ Amputations are often performed due to the damage of the limb involved. They also offer the benefit of being quicker surgeries and can potentially reduce blood loss and operative time, especially in the setting of necrotizing fasciitis that has had overwhelming tissue destruction. ${ }^{[22]}$

After management of the patient's vitals, multiple debridements, careful wound handling, and antimicrobial treatments the patient will ideally recover and the infection will no longer be present. To aid along with the healing management of these complex patients, hyperbaric oxygen therapy has been attempted. Results in terms of its morbidity and mortality benefit have been controversial over the years. One study endorsed the morbidity and mortality benefit stating that through its use it had a mortality difference of $66 \%$ down to $23 \%$ by using hyperbaric oxygen. ${ }^{[23]}$ However, other studies have suggested otherwise and indicate that more research into its efficacy should be done. ${ }^{[24,25]}$

\section{Case report 1}

A 43-year-old patient with a history of pre-diabetes, multiple previous skin abscesses status post drainage, and renal insufficiency following radio contrast dye that presented to the Emergency Department due to crippling pain in his right arm.

The patient was seen before admission in the ED two days earlier for swelling and erythema of the right lower extremity. On this visit he was given out the door IV clindamycin and discharged on $400 \mathrm{mg}$ clindamycin PO. He endorsed that he adhered to the treatment program but returned two days later due to right upper extremity swelling, erythema, and pain. This pain was inhibiting his activities of daily living. He denied any trauma, animal/human bites/scratches, chills, fevers, night sweats, or recent abscesses in the axilla before the onset of the erythema. His vitals showed an oral temper- 
ature of $97.7 \mathrm{degF}$, a pulse of $103 \mathrm{bpm}$, respiratory rate of 16 breaths per minutes, blood pressure $144 / 81 \mathrm{mmHg}$, and a pulse oximetry of $96 \%$ on room air. On examination the patients showed erythema and induration in the right axilla that was warm and tender to palpation. The right elbow and hand were also warm, erythematous, and tender.

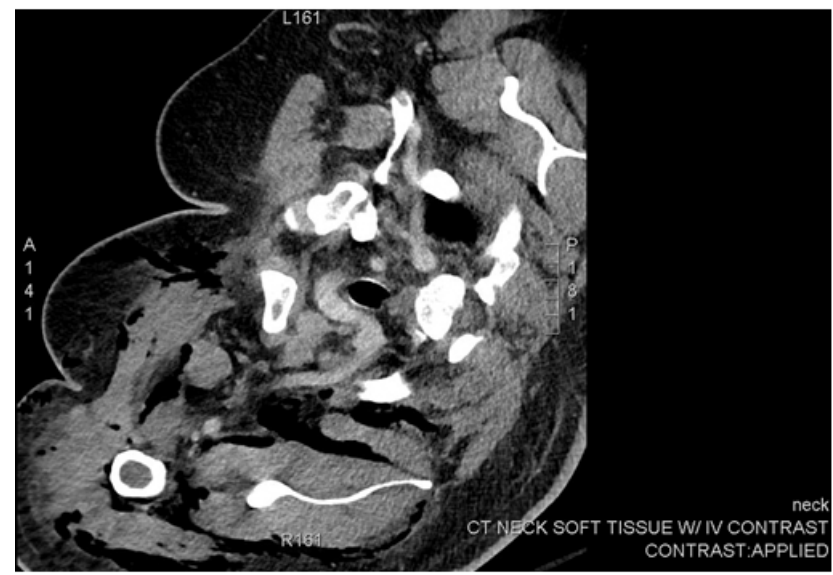

Figure 1. A CT scan performed on admission showing gas and fluid collections in the right upper extremity

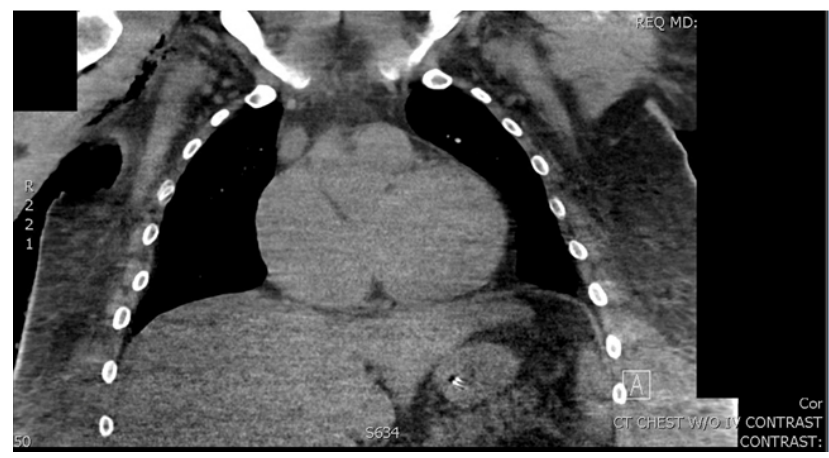

Figure 2. A CT performed a couple of days after the first incision and debridement showing continued presence of gas and fluid collections

Crepitus was noted extending from the right axilla to the posterior superior right arm to the right clavicle. The patient WBC was $19.1 \mathrm{~K} / \mu \mathrm{l}$ comprised of $89 \%$ polynucleated neutrophils with an absolute neutrophil count of 18.53 $\mathrm{K} / \mu \mathrm{l}$. His band neutrophils were $8 \%$. H\&H was normal and C-reactive protein was $439.0 \mathrm{mg} / \mathrm{L}$. The patient was diagnosed with sepsis and showed signs concerning for a gas producing bacterium as crepitus was detected. Broad-spectrum antibiotics of vancomycin, zosyn, and clindamycin were initiated and blood culture obtained. He was quickly taken to the operating room where a right upper extremity incision and debridement was performed along with tissue culture collection. Shortly after the procedure he required intubation due to declining oxygen saturation. CT imagine was Published by Sciedu Press performed which showed continued gas presence from the bacterium as seen in Figures 1 and 2.

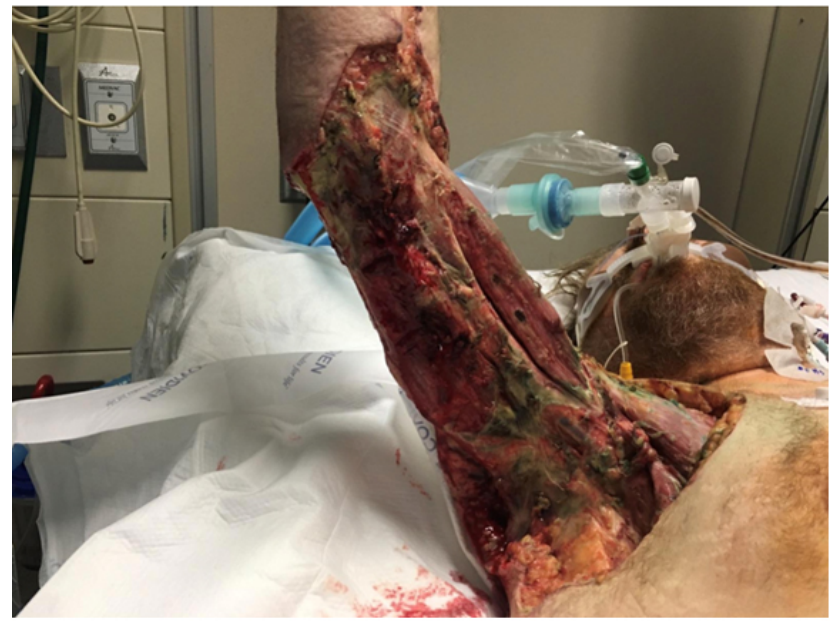

Figure 3. Scheduled re-examination of arm 24 hours after first debridement showed continued wound infection

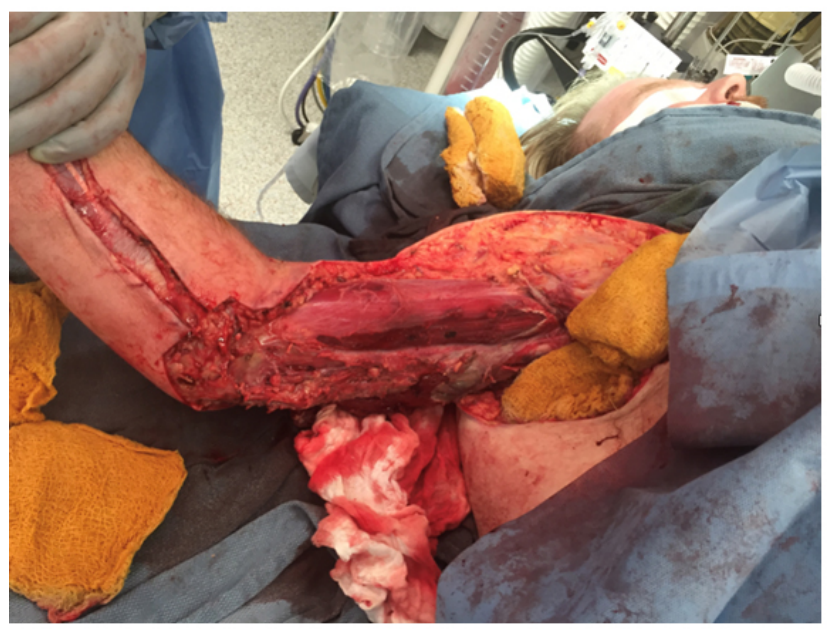

Figure 4. Post debridement of the upper right extremity

The patient was diagnosed with HIV and IVIG was administered with antiretroviral treatments being withheld at this time. His culture began to show gram positive cocci with gram stain and a bedside debridement was performed. His wound cultures were found to have lactococcus and klebsiella pneumonia. Infectious disease recommended treatment to unasyn and flagyl to cover for klebsiella and lactococcus. With continued trips to the operating room for incision and drainage treatments his wound progressively began to improve as seen in comparison between Figure 3 and Figure 4. Subsequently CT imagine was also performed which showed no more signs of any gas forming bacteria (see Figure 5).

Due to persistent fevers infectious diseases switched his antibiotics to Vancomycin and Zosyn. Further wound cultures 
returned showing Serratia as another microbe involved in the wound. At this time his antibiotic coverage was changed to Rocephin to cover Klebsiella and the Serratia and Flagyl to continue coverage for anaerobic bacteria. This antibiotic coverage showed promise and the patient's fevers and elevated

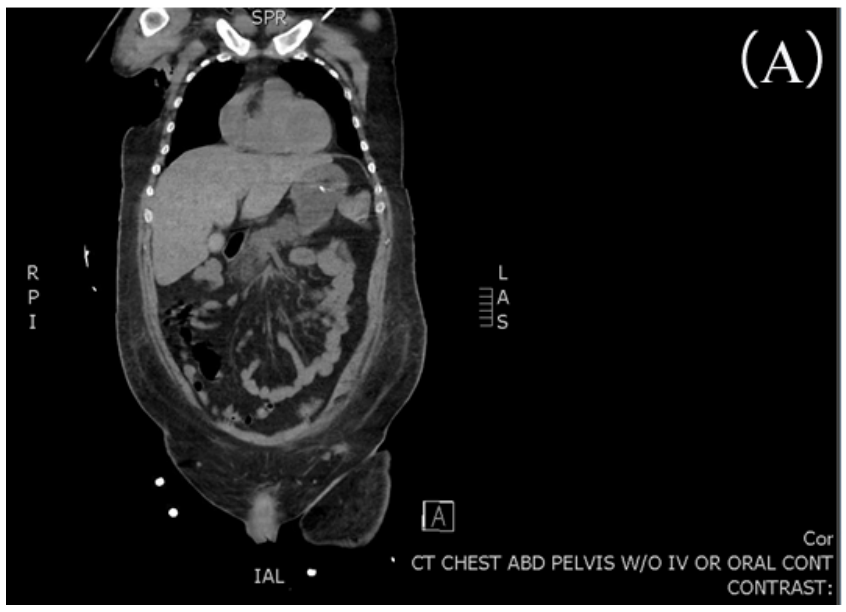

WBC resolved. Plastic surgery began to debride the wound further and placed a split thickness skin graft harvested from his left lower extremity. He was started on his antiretroviral therapy for his HIV infection and the patient was eventually discharged to a health and rehabilitation facility.

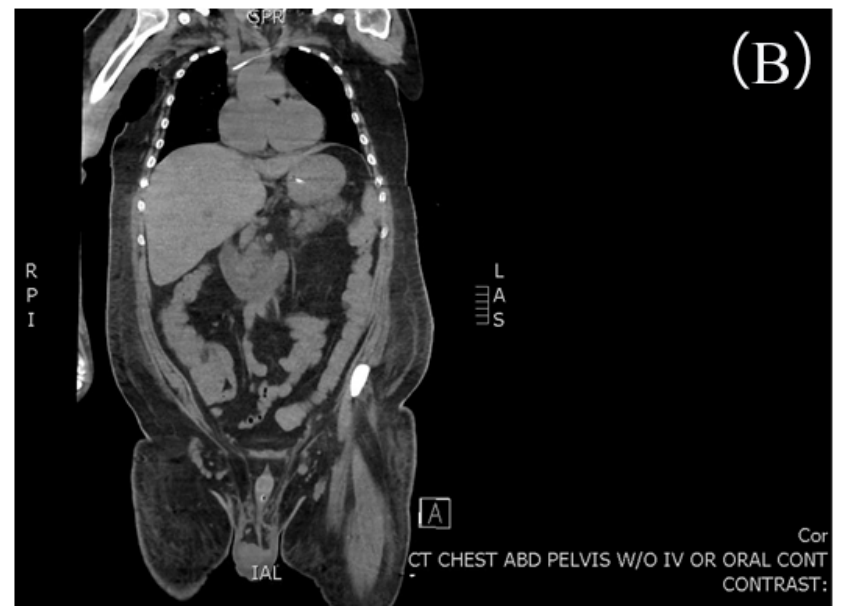

Figure 5. Nine days after admission

Air and fluid collections are no longer present. Also present are signs of the wound debridement

\section{Case report 2}

A 71-year-old African American Male with a history of congestive heart failure, coronary artery disease, atrial fibrillation, peripheral artery disease, COPD, and hyperlipidemia who presented with concerns of increased output from his perianal fistula.

He was on 2.5 L home oxygen due to his COPD. The patient has had the anal fistula for eight months, during which his wife has been able to assist with packing and cleaning it. $\mathrm{He}$ referred to the surgery clinic for evaluation and treatment, he was then scheduled for a EUA and fistulectomy. A week later he is admitted to the emergency department due to in- crease drainage, pain and fever. On admission vitals showed a temperature of $98.2 \mathrm{degF}$, heart rate $113 \mathrm{bpm}$, respiratory rate 20 breaths/minute, blood pressure 110/65 $\mathrm{mmHg}$, and $\mathrm{SpO}_{2} 93 \%$. On examination he was alert and oriented $\times 4$. External rectal exam showed a foul smelling serosanguinous non-purulent drainage from the anus with no signs of an external lesion. White blood cell count was $22.3 \mathrm{~K} / \mu \mathrm{l}$ with an absolute neutrophil count of $20.96 \mathrm{~K} / \mu \mathrm{l}$. A CT scan (see Figure 6) of the chest, abdomen, and pelvis was obtained with signs worrisome for a gas forming soft tissue infection within the medial adductor muscle compartment of the upper thigh and hip joints, left rectus sheath, base of the penis, and suprapubic area.
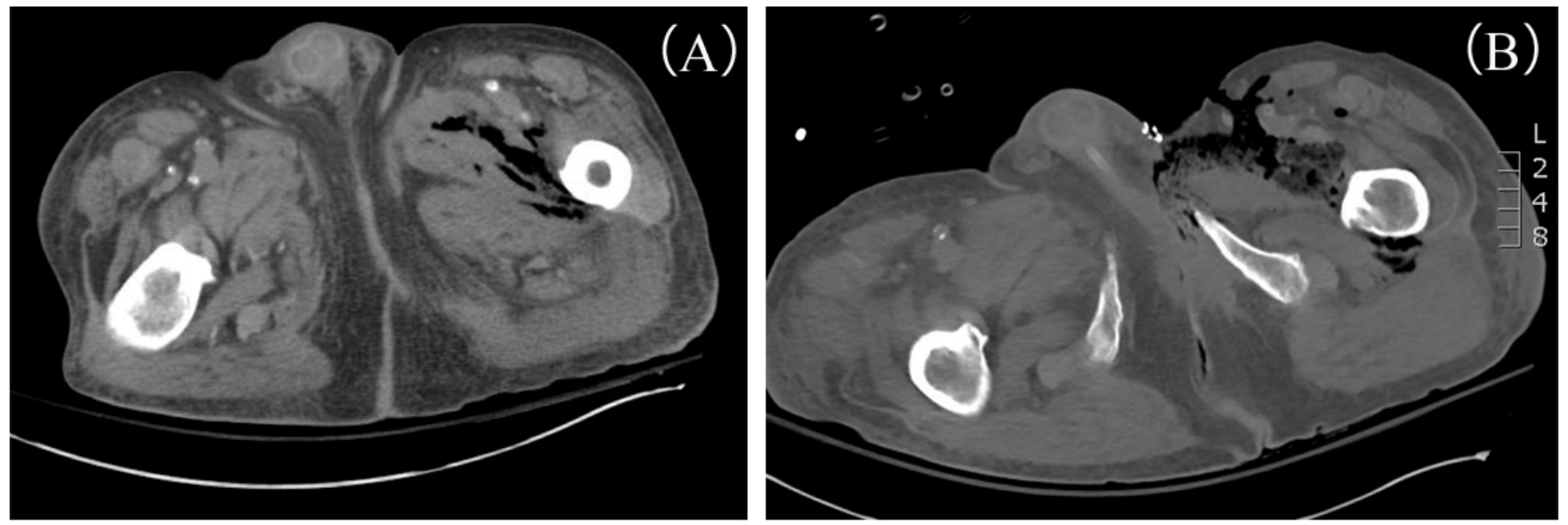

Figure 6. CT scans of the pelvis showing concerning signs for a gas forming soft tissue infection 
Patient at this time was scheduled to have emergent debridement done in the OR. The procedure was performed and a specimen was obtained from he left medial deep thigh tissue. Following the procedure the patient was admitted to the STICU and remained intubated secondary to septic shock. Vancomycin, Zosyn, and clindamycin were started immediately for the infection. The following day the patient went back into the OR to have further debridement of a left iliopsoas abscess in conjunction with orthopedics for pubic bone osteomyelitis.

Over the next week the patient underwent two more wound debridements and a tracheostomy tube was placed for respiratory failure. Wound cultures came back positive for Bacteroides fragilis, Streptococcus anginosus, and Prevotella species. Antibiotics were de-escalated to Unasyn and scheduled for six weeks to adequately treat his osteomyelitis. With him improving on his current treatment plastic surgery began to be involved with commencement of wound closure. The patient had an end colostomy performed to facilitate perineal wound healing. Over the next few weeks the patient's conditions improved and he was transferred to a rehab facility. Plastic surgery followed up with the patient and finished closure of the wound.

\section{Case report 3}

A 44-year-old Caucasian female with diabetes mellitus type 2, hypertension, dyslipidemia, hypercholesterolemia and coronary artery disease that presented with complaints of dyspnea with significant cellulitis of the left side of the neck.

This patient had untreated dental caries and multiple oropharyngeal abscesses on admission. Patient was dyspneic at rest and immediately transferred to MICU for endotracheal intubation and IV antibiotics. She was admitted with a white blood cell count of $16.1 \mathrm{~K} / \mu \mathrm{l}$ and imaging of head and neck was done to better characterize the lesions. The decision was made to proceed to the OR for debridement and irrigation of neck wounds and abscesses necessitating the placement of a wound vacuum (see Figures 7 and 8). Diagnosis made at this time was consistent with Ludwig's Angina, which subsequently evolved, into cervicofacial necrotizing fasciitis.

Intraoperative cultures were positive for Candida albicans and dubliensis species in addition to Klebsiella. She was initially started on Rocephin, Flagyl and Fluconazole, however given culture results, was switched to Anidulafungin. Tracheal secretions were cultured and positive for Pseudomonas. Given possibility of Pseudomonas tracheobronchitis, Cefepime was started prophylactically.

In following weeks, Plastic Surgery performed numerous debridements for recurring necrosis and dehiscence of wound margins. ${ }^{[11]}$ Hyperbaric oxygen therapy was given to aid the healing process. A left pectoralis musculocutaneous flap was used for closure of the defect, which now included the face, neck and upper chest. The left lateral and medial pectoral nerves were transplanted with the flap for a total of $55 \mathrm{~cm}^{2}$. After several weeks in the ICU, she was eventually discharged to a rehab facility.

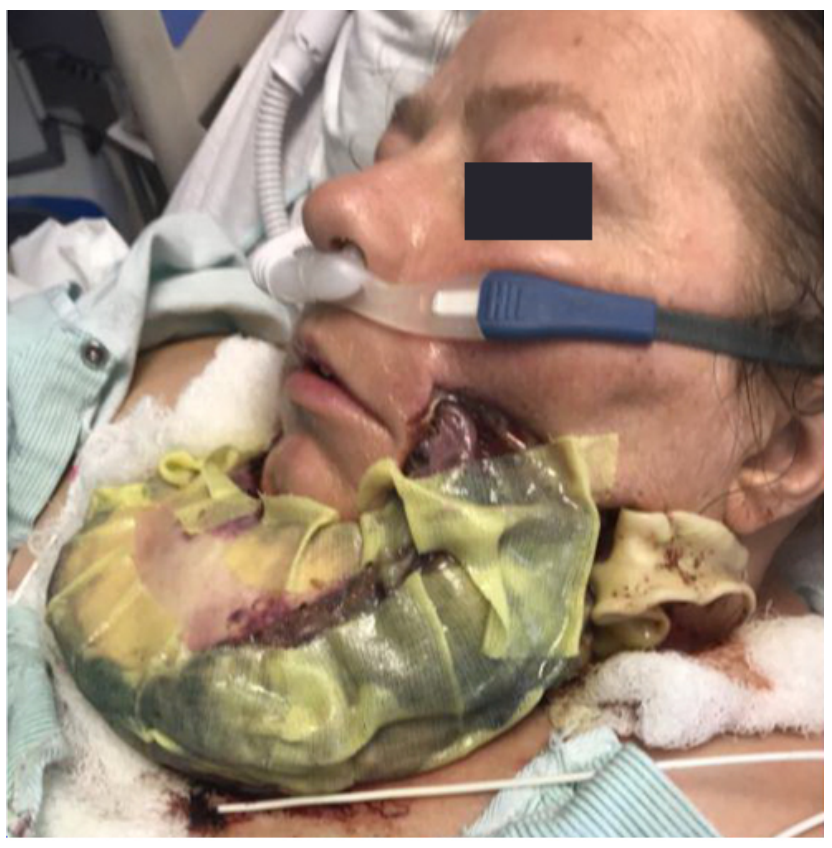

Figure 7. Wound bag in place after debridement in OR was performed

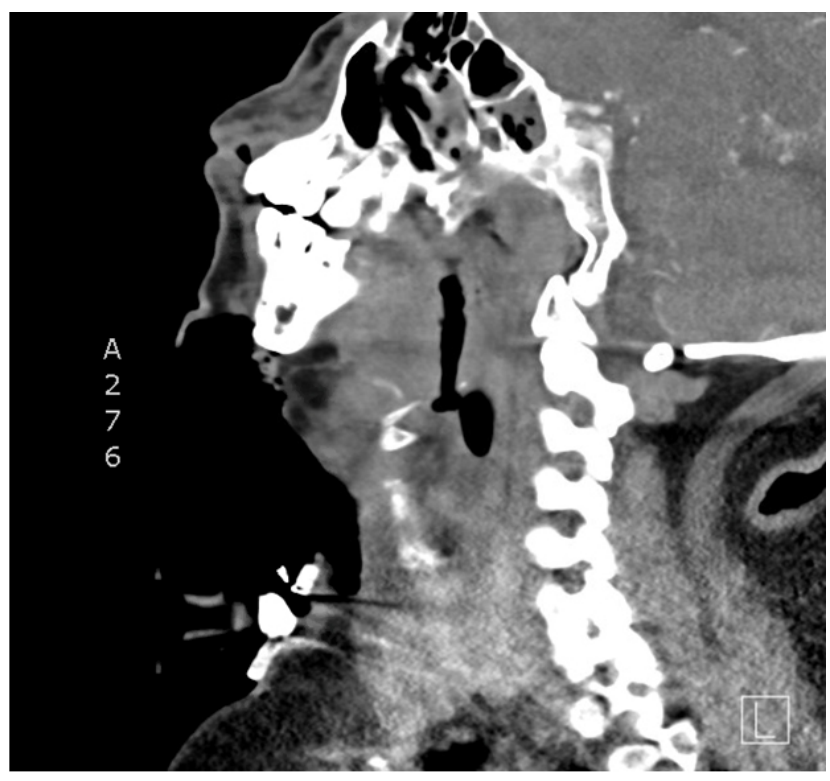

Figure 8. CT scan performed after debridement with placement of tracheostomy and wound bag 


\section{DisCusSION}

As seen in these three cases necrotizing fasciitis is a serious infection that requires rapid response by a surgical team. With the clinical suspicion each patient was started immediately on IV antibiotics and taken to the OR for debridement. Additionally, tissue cultures were collected for further classification of the offending organism and susceptibility testing was performed in order to provide the most targeted antimicrobial treatment. As the process of treatment continued the patients returned to the OR multiple times for repeated debridements and washings. Each patient was cared for in an ICU setting where close monitoring and management of their clinical condition could be performed. Following the surgical management of each patient's infection the services of plastic surgery were necessary to restore the affect area to a respectable form.

Of interest all three patients shared a previous diagnosis of diabetes mellitus, which has been shown in literature to increase the risk of limb loss morbidity along with its association with polymicrobial and K. Pneumonia necrotizing fasciitis infections. ${ }^{[23]}$ All three patients had polymicrobial infections with Patient 2 being unique with not having K. Pneumonia among the microbes cultured. Patient 3 was also unique in that her infection was complicated by the presence of candida species among the K. pneumonia. She also was the only patient of the three to receive hyperbaric oxygen therapy to help promote wound healing.

In classifying the three patients necrotizing fasciitis, patient 1 and 2 were among Class 1 . Patient 1 and 2 both have a past medical history that increases the chances for a Class I infec- tion with Patient 1 being immunocompromised (HIV) and Patient 2 having diabetes. Patient 3 was unique in that she was in the class IV rating since her infection was complicated with fungal species. Due to the presence of fungal specimens in the wound this patients clinical treatment required the addition of antifungals along with the typical management with antibiotics for Class I organisms.

Patient 1 HIV status likely may have contributed to his noteworthy presentation in that he didn't have any predisposing trauma or nidus to initiate the infection. The other two patients both had an open wound that provided access for infection with Patient 2 having an anocutaneous fistula and Patient 3 had recurrent dental carries. With Patient 1's HIV status he was also the only patient who had to be placed on IVIG to assist his body in combating the organisms infecting it.

Patient 2 due to his COPD and diminished lung capabilities weaning off the ventilator posed to be a challenge over the coming weeks recurring in multiple attempts and failures. Ultimately, through the use of careful weaning technique he was able to be extubated.

Each patient presented had a different location affected and a slightly different clinical course. However, even with the differences the overall layout for treatment remained constant. Urgently, the patient required debridement of the affected area and then careful management was tailor specifically to each patients needs moving forward.

\section{CONFlicts of InTERest Disclosure}

The authors declare they have no conflicts of interest.

\section{REFERENCES}

[1] Leitch HA, Palepu A, Fernandes CM. Necrotizing fasciitis secondary to group A streptococcus. Morbidity and mortality still high. Canadian Family Physician. 2000; 46(7): 1460-1466. PMid: 10925760.

[2] Hakkarainen TW, Kopari NM, Pham TN, et al. Necrotizing soft tissue infections: review and current concepts in treatment, systems of care, and outcomes. Current Problems in Surgery. 2014 Aug; 51(8) 344-62. PMid: 25069713. https://doi.org/10.1067/j.cpsu rg. 2014.06.001

[3] Hakkarainen TW, Kopari NM, Pham TN. Necrotizing soft tissue infections: Review and current concepts in treatment, systems of care, and outcomes. Current Problems in Surgery. 2014 August; 51(8): 344-362. PMid: 25069713. https://doi.org/10.1067/j.cpsu $\mathrm{rg} .2014 .06 .001$

[4] Stevens DL, Bryant AE. Necrotizing Soft Tissue Infections. The New England Journal of Medicine. 2017 December 7; 377: 2253-2265 PMid: 29211672. https://doi.org/10.1056/NEJMra1600673

[5] Wong CH, Wang YS. The Diagnosis of Necrotizing Fasciitis. Current Opinion in Infectious Disease. 2005 April; 18(2): 101-6.
PMid: 15735411. https://doi.org/10.1097/01.qco.00001 60896.74492.ea

[6] Stevens DL, Bisno AL, Chambers HF, et al. Practice guidelines for the diagnosis and management of skin and soft tissue infections: 2014 update by the infectious diseases society of America. Clinical Infectious Disease: an official publication of the Infectious Disease Society of America. 2014 July 15; 59(2): 147-59.

[7] Misiakos EP, Bagias G, Patapis P, et al. Current Concepts in the Management of Necrotizing Fasciitis. Frontiers in Surgery. 2014 September 29; 1: 36. PMid: 25593960. https://doi.org/10.3 389/f surg. 2014.00036

[8] Wong CH, Khin LW, Heng KS, et al. The LRINEC (Laboratory Risk Indicator for Necrotizing Fasciitis) score: a tool for distinguishing necrotizing fasciitis from other soft tissue infections. Critical Care Medicine. 2004 July; 32(7): 1535-41. PMid: 15241098. https://doi.org/10.1097/01.CCM.0000129486.35458.7D

[9] Neeki MM, Dong F, Au C, et al. Evaluating the Laboratory Risk Indicator to Differentiate Cellulitis from Necrotizing Fasciitis in the Emergency Department. The Western Journal of Emergency 
Medicine. 2017 June; 18(4): 684-689. PMid: 28611889. https : //doi.org/10.5811/westjem.2017.3.33607

[10] Becker M, Zbären P, Hermans R, et al. Necrotizing fasciitis of the head and neck: role of CT in diagnosis and management. Radiology. 1997 February; 202(2): 471-6. PMid: 9015076. https: //doi.org/10.1148/radiology.202.2.9015076

[11] Castleberg E, Jenson N, Dinh VA. Diagnosis of Necrotizing Faciitis with Bedside Ultrasound: the STAFF Exam. West Journal Emergency Medicine. 2014 February; 15(1): 111-113. PMid: 24578776. https://doi.org/10.5811/westjem.2013.8.18303

[12] Ali SZ, Srinivasan S, Peh WCG. MRI in necrotizing fasciitis of the extremities. The British Journal of Radiology. 2014 January; 87(1033): 20130560. PMid: 24288403. https://doi.org/10.1 259/bjr. 20130560

[13] Schmid MR, Kossmann T, Duewell S. Differentiation of necrotizing fasciitis and cellulitis using MR imaging. American Journal of Roentgenology. 1998 March; 170(3): 615-20. PMid: 9490940. https://doi.org/10.2214/ajr.170.3.9490940

[14] Wall DB, Klein SR, Black S, et al. A simple model to help distinguish necrotizing fasciitis from nonnecrotizing soft tissue infection. Journal of American College of Surgery. 2000 September; 191(3): 227-31. https ://doi.org/10.1016/S1072-7515(00)00318-5

[15] Kalaivani V, Hiremath BV, Indumathi VA. Necrotising soft tissue infection-risk factors for mortality. Journal of Clinical and Diagnostic Research. 2013 Aug; 7(8): 1662-5.

[16] Sartelli M, Malangoni MA, May AK, et al. World Society of Emergency Surgery (WSES) guidelines for management of skin and soft tissue infections. World Journal of Emergency Surgery. 2014; 9(1): 57. PMid: 25422671. https ://doi .org/10.1186/1749-7 922-9-57

[17] Van Stigt SFL, de Vries J, Bijker JB, et al. Review of 58 patients with necrotizing fasciitis in the Netherlands. World Journal of Emergency Surgery. 2016 May 27; 11: 21. PMid: 27239222. https://doi.org/10.1186/s13017-016-0080-7
[18] Majeski J, Majeski E. Necrotizing fasciitis: improved survival with early recognition by tissue biopsy and aggressive surgical treatment. Southern Medical Journal. 1997 Nov; 90(11): 1065-8. PMid: 9386043. https://doi.org/10.1097/00007611-19971 1000-00001

[19] Shah AK, Kumar NB, Gambhir RP, et al. Integrated clinical care pathway for managing necrotizing soft tissue infections. Indian Journal of Surgery. 2009 Oct; 71(5): 254-7. PMid: 23133168 https://doi.org/10.1007/s12262-009-0076-6

[20] Glass GE, Sheil F, Ruston JC, et al. Necrotising soft tissue infection in a UK metropolitan population. Annals of the Royal College of Surgeons of England. 2015 Jan; 97(1): 46-51. PMid: 25519266. https://doi.org/10.1308/003588414X14055925058553

[21] Eray IC, Alabaz O, Akcam AT, et al. Comparison of Diverting Colostomy and Bowel Management Catheter Applications in Fournier Gangrene Cases Requiring Fecal Diversion. Indian Journal of Surgery. 2015 Dec; 77(Suppl 2): 438-41. PMid: 26730041. https://doi.org/10.1007/s12262-013-0868-6

[22] Angoules AG, Kontakis G, Drakoulakis E, et al. Necrotizing fasciitis of upper and lower limb: a systematic review. Injury. $2007 \mathrm{Dec} ; 38$ Suppl 5: S19-26. PMid: 18048033. https://doi.org/10.1016/ j.injury.2007.10.030

[23] Riseman JA, Zamboni WA, Curtis A, et al. Hyperbaric oxygen therapy for necrotizing fasciitis reduces mortality and the need for debridements. Surgery. 1990 November; 108(5): 847-50. PMid: 2237764.

[24] Shupak A, Shoshani O, Goldenberg I, et al. Necrotizing fasciitis: an indication for hyperbaric oxygenation therapy? Surgery. 1995 November; 118(5): 873-8. https ://doi .org/10.1016/S0039-6 060 (05) 80278-8

[25] Massey PR, Sakran JV, Mills AM, et al. Hyperbaric oxygen therapy in necrotizing soft tissue infections. The Journal of Surgical Research. 2012 September; 177(1): 146-51. PMid: 22487383. https://doi.org/10.1016/j.jss.2012.03.016 